\title{
Des agriculteurs, en chemin vers l'écologie, éprouvent le paysage. Les projets de plantations agroforestières en Haute-Garonne.
}

\section{Farmers shape their own ecological landscape by planting trees, case study in South West of France »}

\author{
Mathilde Rue ${ }^{1}$
}

${ }^{1}$ Laboratoire Interdisciplinaire Solidarités, Sociétés, Territoires (LISST) - équipe Dynamiques Rurales (UMR CNRS 5193, Université de Toulouse et ENSFEA), associée au Laboratoire de Recherche en Projet de Paysage, Versailles (LAREP, ENSP). Bourse de recherche Fondation de France, mathilde.rue@univ-tlse2.fr

Ce travail de recherche est soutenu par la Fondation Edouard et Geneviève Buffard sous l'égide de la Fondation de France.

RÉSUMÉ. L'agriculteur intervient sur son paysage quotidien. A l'heure où les questions d'écologie s'affirment, certains agriculteurs choisissent d'implanter quelques centaines d'arbres sur leurs terres, les associant à une autre culture ou des animaux (agroforesterie intraparcellaire). Ce sont aussi des personnes en reconversion, qui choisissent un nouveau mode et lieu de vie. Que dessinent-ils? A partir d'une étude menée en Haute-Garonne, nous observons que les agriculteurs agroforestiers cherchent à élaborer leur paysage en y intégrant des rêves et leur souci écologique. Le paysage devient le canal et le lieu d'expérimentation et d'expression d'une construction écologique du rapport au monde. Avec une méthode empirique et compréhensive, aidée des outils du projet de paysage, nous verrons comment aspirations paysagères et écologie ont à voir ensembles.

ABSTRACT. Farming practices shape landscape. Farmers experience it in their everyday routine (Henry, 2012) and landscape is related to the living ("habiter", Besse, 2009). Modern agroforestry which means land-use systems where woody perennials are deliberately integrated with crops and/or animals on the same parcel is encouraged for ecological reasons. How do farmers deal with it? Our study shows that agroforestry also could be chosen for benefitting the living environment or to communicate with the neighborhood (Rue, 2018). Agroforestry is an effective method of involving them in a design process on their territory. We have analyzed the adoption of Agroforestry by looking at designs, diversity with regard to farmers' life course trajectory and its impacts at the Landscape scale. The aesthetic and affective (biographic) aspects of these projects need to be considered more and related to ecological values.

MOTS-CLÉS. élaboration du paysage, agriculteurs, paysage matrice, paysage référent, paysage projeté, agroforesterie, arbre, habiter, relation paysagère.

KEYWORDS. landscape planning, farmers, agroforestry, tree, to live in, landscaped relationship.

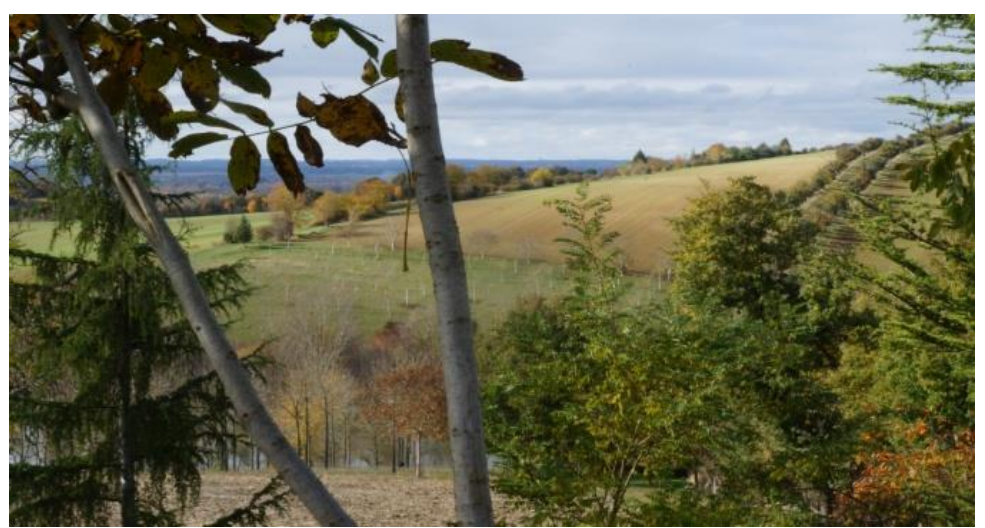

Figure 1. Chez Roland, vue sur des plantations diverses (C) Mathilde Rue 
Le chemin de l'écologie, en agriculture, conduit à repenser les pratiques de la ferme. Nous verrons à travers l'exemple de l'adoption de système agroforestier intraparcellaire que s'engage, en même temps, un processus d'élaboration paysagère.

Par définition, l'agriculteur « entretient», « soigne», «cultive ». Ces mots ont pour origine commune le latin colere qui signifie aussi «habiter». «Nous sommes habitant, au participe présent, dans nos activités quotidiennes et exceptionnelles, nos gestes, nos habitudes, nos façons différentes d'être présents à l'espace et de nous y conduire, voire de nous laisser imprégner par les lieux dans lesquels nous nous tenons régulièrement»(Besse, 2015, p10). L'agriculteur est un habitant particulier qui dans son travail construit et façonne son environnement. Certains d'entre eux (au même titre que d'autres citoyens) aspirent à une révision de leur rapport à la Terre. A la fois, pour «s'arranger» un cadre et un mode de vie selon leurs goûts et leurs valeurs (pour eux-mêmes) et à la fois parce que le fruit de leur activité est amené à être partagé avec le reste de la société qu'il s'agisse de nourriture, de territoire, de nature. Dans notre intérêt pour les liens entre agriculture et paysage (Henry, 2012) nous questionnons la construction de l'habiter par les agriculteurs à travers leur décision d'installer un système d'agroforesterie intraparcellaire.

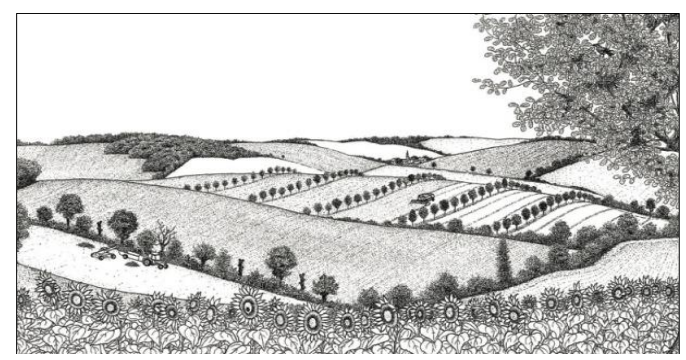

Figure 2. Des agroforesteries et la forme intraparcellaire. (C) David Dellas, Arbres et Paysages 32

L'agroforesterie intraparcellaire est un mode agricole qui associe sur une même parcelle des alignements d'arbres à une autre production afin de garantir un ensemble de bénéfices (préservation des sols, maintien de la biodiversité, qualité de l'eau, bien-être animal, etc.). Dans le même temps, l'agroforesterie procède d'une redéfinition spatiale que nous trouvons opportun d'interroger (figure 2). Elle est à la fois une transformation de l'espace physique mais aussi des représentations sociales. Autrement dit, un agriculteur qui plante des arbres change le paysage, cela se voit et veut dire quelque chose. Nous faisons l'hypothèse que ces personnes s'orientent vers l'agroforesterie car elles y perçoivent la possibilité d'une mise en paysage de leurs aspirations de vie, de leur rapport au monde. Nous postulons - qu'au-delà d'enjeux agronomiques, économiques, environnementaux - il y a avec l'agroforesterie, une projection de soi dans une manière choisie d'habiter le monde. Nous parlerons de «l'habiter » comme la mise au travail de la relation paysagère de l'agriculteur. Cette relation au sens d'Augustin Berque lie indissolublement les objets et le sujet: «Un paysage fonctionne à la fois comme empreinte et comme matrice : empreinte, parce qu'il exprime des façons de faire et des façons de voir qui lui sont antérieures ; et matrice, parce qu'il informe à son tour des façons de voir et des façons de faire qu'exprimeront ultérieurement d'autres paysages.", (Berque, 1987). L'agroforesterie intraparcellaire est l'occasion d'activer ces flux de relations et de transformer le paysage (tant représenté que vécu). La majorité des agriculteurs agroforestiers du département sont en agriculture biologique ou bien en non travail du sol: en quoi le dispositif spatial arboré qu'est l'agroforesterie offre-t-il la possibilité d'une re-composition du paysage, en résonance avec des projets de vie et de travail aux valeurs écologiques ? Comment habite-on, «faiton système » avec son environnement dans le cadre de la ferme ? Quels sont les imaginaires, les pratiques (agricoles, habitantes), les gestes, les formes produites, les temporalités et les lieux 
engagés dans une posture paysagère ayant le souci écologique ? Quelles sont ces formes projetées, mais aussi les ressources dont se nourrissent les agriculteurs pour concevoir leur projet?

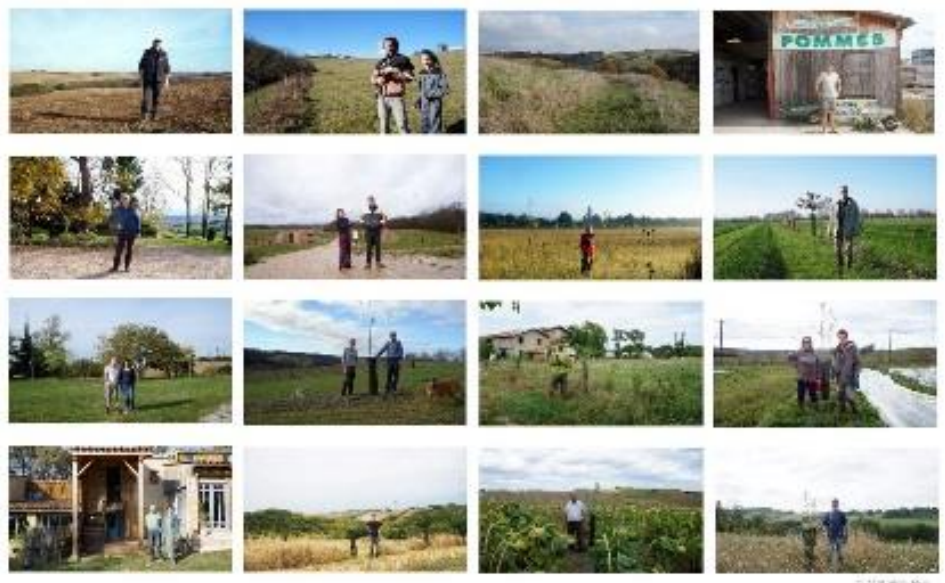

Figure 3. Les seize agriculteurs de l'enquête principale. ( Mathilde Rue

Notre méthode d'enquête s'est construite au fil de l'expérience des terrains, dans une approche principalement sensible et compréhensive. Mené au sein de 16 fermes de Haute-Garonne, l'ensemble des dispositifs (observation, entretiens conversationnels, relevés sons et images, «observatoire filmique des paysages ») ont été reconduits dans le temps (2016-2019). Cette démarche croisant les données et les regards a permis d'étudier la relation paysagère des agriculteurs dans leurs diverses implications (attendues comme inattendues) et dans leur évolution.

Il est pour l'heure proposé d'organiser cette lecture selon les étapes identifiées du processus de projet agroforestier. Pour chacune d'elle, nous envisagerons la participation du paysage à l'élaboration.

\section{Le processus d'élaboration paysagère agroforestière en 6 étapes}

\section{Etape 1. Emergence de l'idée.}

Comment naît le projet d'agroforesterie ? L'idée agroforestière a différentes sources. Pour certains agriculteurs, elle vient d'un paysage expériencé, vécu, perdu. Puisé dans l'histoire personnelle, il est un référent chargé d'émotions, d'affects, d'esthétiques et de pratiques. Il correspond à un moment donné et à un mode de vie particulier. C'est le cas de Sylvain, ancien berger qui dit avoir essayé de ramener, sur sa ferme actuelle située à $45 \mathrm{~min}$ au sud de Toulouse, les Pyrénées où il garda des moutons (figure 4).

Pour d'autres, c'est par une entrée théorique qu'ils abordent l'agroforesterie. Néophytes se formant à l'agriculture, c'est par la découverte du mot, la lecture d'un livre ou encore la participation à une conférence qu'ils s'y intéressent. Ils en feront ensuite une modalité de base de leur système agraire. Cette compréhension du concept agroforestier est véhiculée par certains réseaux (agroécologie, permaculture, agroforesterie...) et donne à adhérer à une représentation holistique et systémique du milieu (figure 5).

D'autres encore, cette fois expérimentateurs de longue date en agriculture, rencontrent cette pratique chez leurs pairs. Situés dans une démarche d'adaptation générale de leur système aux enjeux de la durabilité, ils envisagent l'agroforesterie comme une pratique supplémentaire à tester (figure 6). 

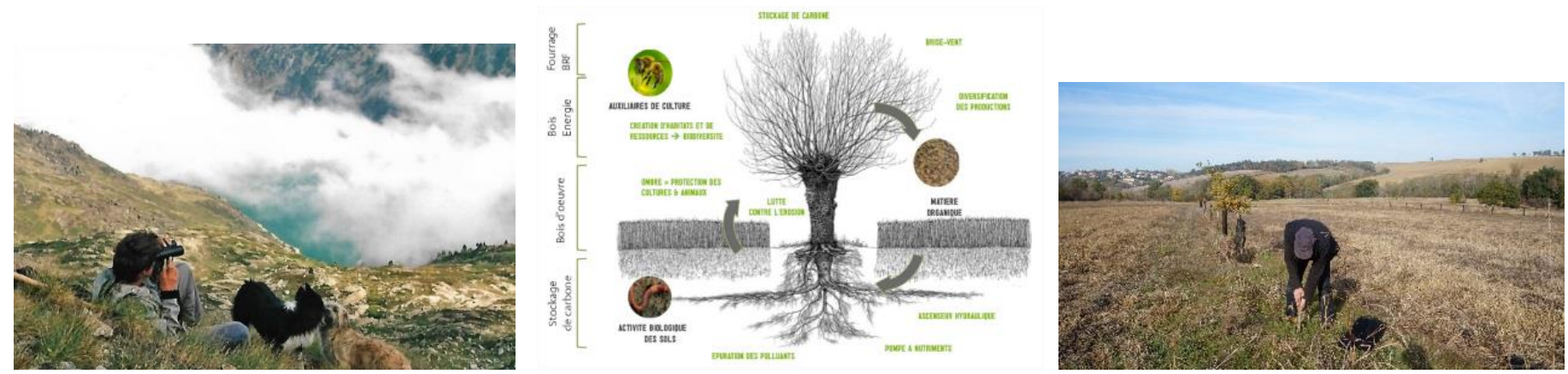

Figure 4. Un paysage expériencé et perdu. @ privée de l'agriculteur

Figure 5. Une théorie qui fait sens. (C) Association Française d'Agroforesterie

Figure 6. Bruno dans sa parcelle plantée en 2015, (C) Mathilde Rue

Ces trois points de départ principaux constituent des ressources-modèles pour le projet en construction : ils participeront à l'élaboration de la parcelle agroforestière en tant que " paysages référents » (Rue, 2018).

L'idée des plantations s'est imposée, la prochaine étape est de choisir le lieu d'installation.

\section{Etape 2. Installation/Choix du lieu.}

«Installation» aura ici un double sens : tout d'abord comme « installation agricole » (la recherche d'une ferme), puis comme l'insertion dans l'unité agricole de la parcelle elle-même. En effet, au moment de l'engagement dans la démarche agroforestière, nombreux sont les agriculteurs encore sans terres : plus de la moitié d'entre eux sont 'hors cadre familial' et cherchent d'abord un lieu où vivre et pratiquer leur nouveau métier. Les personnes de ce premier groupe portent une attention particulière à leur «paysage d'accueil» et ont des attendus en terme de formes et de représentations. Chantal est maraîchère bio avec son époux, Franc. Elle raconte : "On cherchait la ferme de nos rêves, c'est-à-dire pas trop petite, pas trop grande, de quoi cultiver, des bois, un ruisseau, enfin bon si vous voulez c'est un lot de critères qu'on a réussi à rassembler ici. Ça fait un ensemble qui a une certaine cohérence » (figure 7). Nous avons pu relever à plusieurs reprises cette envie d'investir un lieu aux composantes diversifiées, porteur d'un imaginaire bucolique, préservé, paisible : un microcosme.

Dans la seconde situation où les agriculteurs sont installés depuis longtemps et sur la ferme familiale, nous avons pu constater que les critères de choix de la parcelle s'inscrivent dans une filiation paysagère avec l'histoire des lieux. Par-là, les enfants repreneurs poursuivent l'arrangement du microcosme hérité. Bruno est céréalier pratiquant les techniques culturales simplifiées : "On a planté dans le bas-fond. Là, dans le trou, il y a une source. Mon père avait installé une auge et l'été les vaches passaient toute leur journée au fond du bois. Et le soir quand on revenait de l'école c'était notre boulot de venir chercher les vaches, on descendait de la ferme et puis on allait les chercher, là-bas, au fond, et par le sentier on les ramenait à la maison. Donc les vaches elles ont toujours été "à l'abri", à l'ombre, là-bas... [...] Sinon ici, tout le reste était cultivé, c'était 'MontPelé, y avait pas un arbre!» (figure 8). On peut se demander si le choix précis de la parcelle, dans cette très vaste exploitation, n'est pas orienté par un imaginaire paysager de l'enfance. 

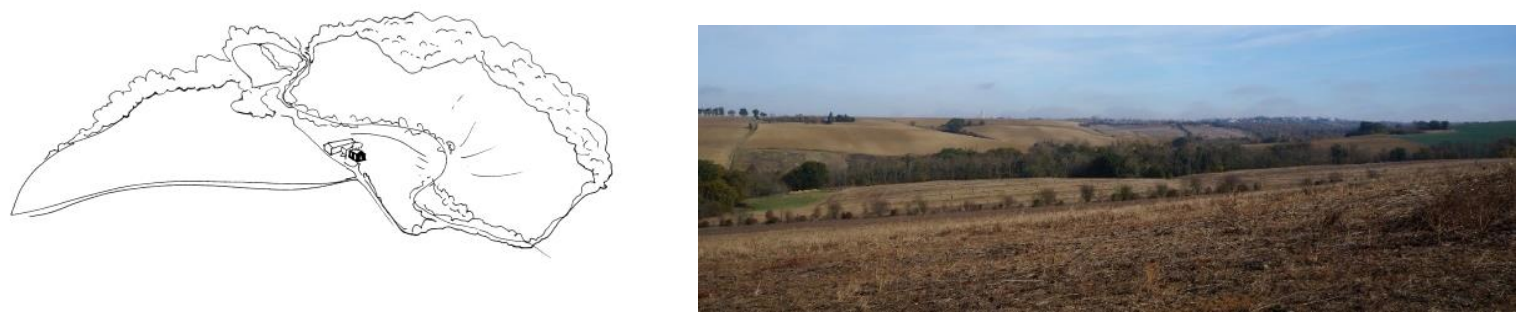

Figure 7. Le choix d'un lieu d'installation qui correspond à des attentes précises. (๐ Mathilde Rue Figure 8. Choisir la parcelle apte à accueillir les arbres sur la ferme familiale, héritée. (c) Mathilde Rue

Sans aucun doute, l'arrivée des arbres intraparcellaires vient soutenir une « installation » plus globale et continue du cadre habité. Dans la plupart des fermes du corpus, il y a eu, au préalable, l'installation de haies et d'allée d'arbres comme chez Éric (figure 9) où celles-ci assurent le rôle de « filtres » entre la route et le siège d'exploitation. Les lignes intraparcellaires, quinze ans plus tard, sont venues renforcer cette enveloppe destinée à délimiter et protéger le lieu de vie (figure10).
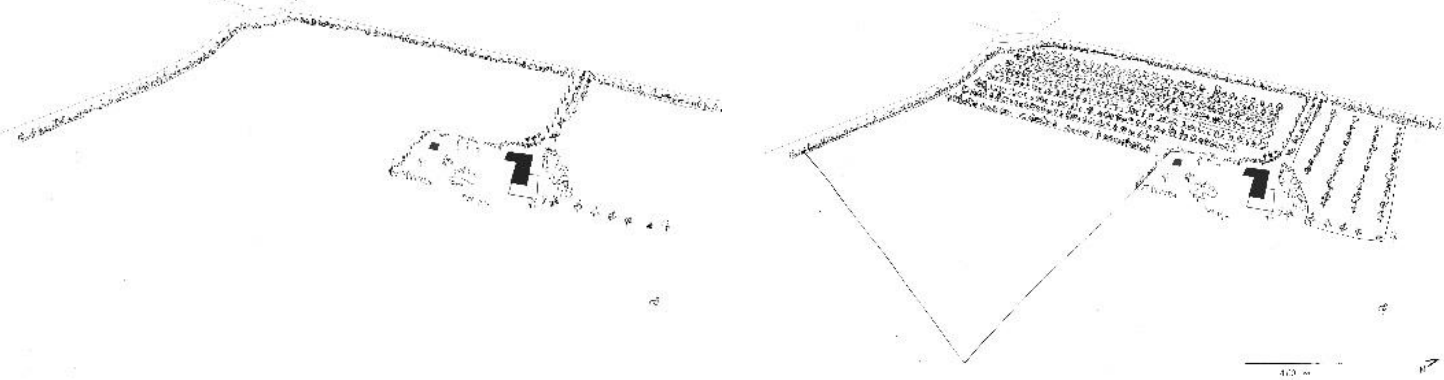

Figure 9. Chez Eric, premières plantations (haie et allée, 2000) (C) Mathilde Rue

Figure 10. Chez Eric, plantations intraparcellaires, 2015 (c) Mathilde Rue

Aux racines des installations de systèmes agroforestiers, si l'ancien berger nous a confié la nécessité pour lui de retrouver une «fragmentation » du paysage comme il l'avait connu dans les montagnes, si Chantal, la maraichère, racontait la composition «idéale » de son unité de ferme, si Bruno, céréalier, convoquait le paysage de son enfance pour justifier l'implantation de ses arbres, concrètement, qu'ont-ils alors planté, et comment ? Au bout du compte, quel paysage ont-ils «mis en terre »?

\section{Etape 3. Conception.}

Cette étape est celle de la formalisation du projet et fait intervenir des ressources et des acteurs extérieurs à l'agriculteur directement impliqués dans le dessin de la parcelle. A ce moment, moyens techniques, règlementations, subventions, expertises mettent le rêve à l'épreuve du réel. De nombreux compromis sont faits : l'orientation des lignes est dictée par rapport à la course du soleil et au sens de travail du sol, les espacements entre les arbres sont dimensionnés par la taille des engins utilisés, les essences sont listées, les productions prédéfinies (figure 11). N'oublions pas que les organismes d'accompagnement des projets se portent garants de la reprise des arbres ainsi que du maintien du potentiel productif de la surface plantée : il faut que ça pousse et que l'agriculteur puisse continuer à cultiver avec ses engins habituels. La réflexion se restreint soudain au périmètre de la parcelle. Ce faisant, nous pouvons déplorer à la fois une lecture moins paysagère lors du diagnostic de site (les questions des points de vue, de l'enchainement des espaces par exemple sont 
évincées de la réflexion), et l'effacement de la participation du référent paysager initial (il y a une perte de la substance paysagère qui alimentait le projet de l'agriculteur).

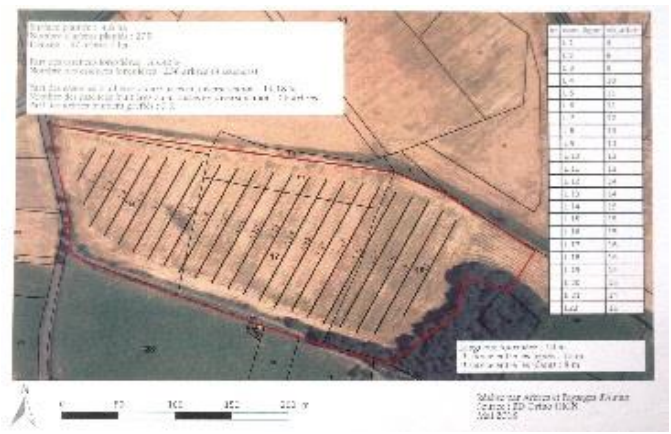

Figure 11. Plan de plantations de Pierre. (C) Arbres et Paysages d'Autan

Néanmoins, une forme de souplesse face à ces normes est mise au travail par certains agriculteurs et leurs appuis, au profit de la singularité de leur vision. Séverine compose un plan de plantations original (figure 12). Elle veut constituer un «Bois Gourmand » comme entité de sa ferme. Sa trame de plantation est asymétrique, elle ne couvre pas toute la parcelle et les essences d'arbres ne sont pas réparties uniformément (à chaque couleur sur le document correspond une essence). Séverine met en place un parcours de volailles. Le couvert arboré peut donc être dense mais pour autant les cabanes (abris mobiles se déplaçant à l'aide de tracteur) auraient pu freiner sa désinhibition dans la composition de la trame plantée. A cet endroit, il nous semble significatif de considérer le référent initial de Séverine. Elle a pour première expérience agroforestière une forêt jardinée au Brésil, système dans lequel les différents étages de cultures sont imbriqués. Or, nous pouvons lire un lien formel entre l'organisation spatiale des arbres de l'agricultrice et ce paysage expériencé, initiatique. La découverte "marquante », hors des cadres et référents locaux ${ }^{1}$ de ce système semble lui avoir donné les moyens d'une prise de liberté dans son projet. Encore différemment, Gabin, jeune maraîcher et éleveur sur moins de 1 ha a lui choisi de répartir ses fruitiers selon différentes modalités d'orientations et de densités, ceci au regard des essences d'arbres et de sa connaissance fine des micro-parcelles (figure 13). Dans les deux présents cas, il peut être observée une prise d'écart par rapport aux modèles issus du monde agricole européen majoritaire. Cette prise de distance d'avec les normes est peut-être à relier au fait qu'ils ne sont pas directement issus du milieu agricole (détachement par rapport à ses codes) et qu'ils ont connu d'autres expériences (possibilité de trouver ressource dans une autre «culture »). Ces deux projets nous offrent d'étayer notre hypothèse initiale et de postuler la mise en route d'une démarche propre à chaque agriculteur pour habiter le monde, chacune orientée par le parcours de $v^{2} e^{2}$. D'autre part, étant hors cadre familial, ces deux agriculteurs n'ont pas de lien d'héritage à ces lieux. Nous pouvons nous demander à titre plus général si nous serions plus libres de transformer un paysage avec lequel nous n'aurions pas de passif généalogique.

\footnotetext{
${ }^{1}$ Le conjoint et le voisinage de Séverine pratiquent l'agriculture de grandes cultures, en champs ouverts.

${ }^{2}$ Au cours de ce processus, force est de constater la participation des modes de réflexions et des outils des uns et des autres dans les formes paysagères produites. Par exemple, Séverine était auparavant conseillère d'entreprise et Gabin ingénieur informaticien. Ils ont su mettre à profit leurs outils numériques pour modéliser et dessiner leur projet eux-mêmes (Excel) et de cette façon, ils ont « gardé la main » sur la conception de la parcelle.
} 

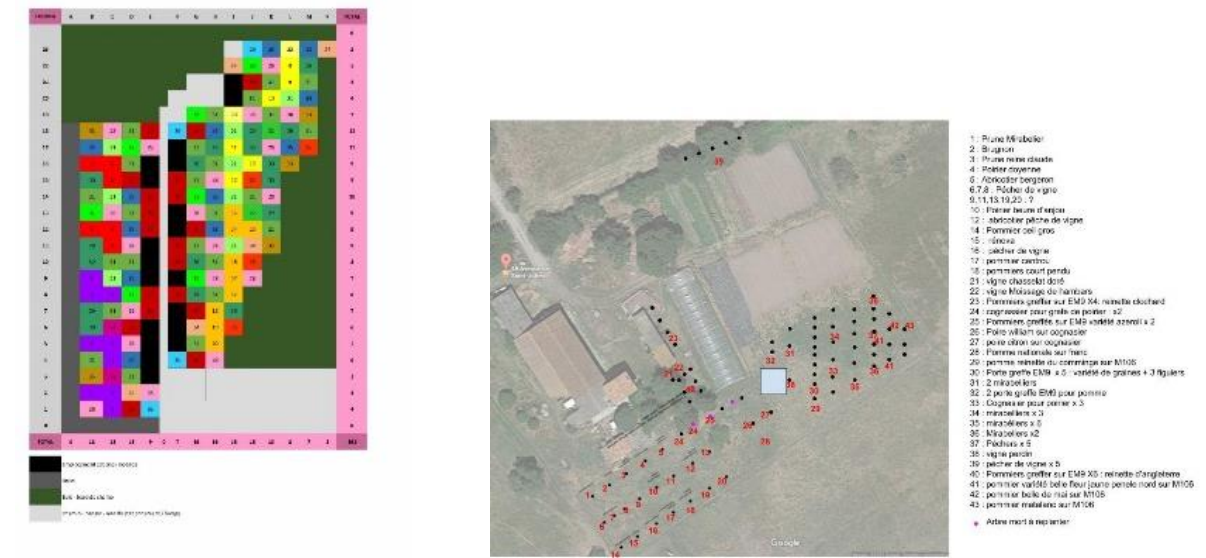

Figure 12. Plan de plantations de Séverine. (C) privée, à l'agricultrice

Figure 13. Plan de plantations de Gabin. (c) privée, à l'agriculteur

\section{Etape 4. Réalisation.}

Le temps de la réalisation in situ re-mobilise l'agriculteur, celui-ci faisant les travaux (en plus d'être au quotidien gestionnaire des espaces de la ferme, il est la plupart du temps maître d'œuvre des nouvelles plantations). Malgré la quantité d'arbres à mettre en terre (souvent plusieurs centaines), et au-delà d'un aspect uniquement pécunieux, les agriculteurs plantent eux-mêmes, voire tout «à la main » pour l'un d'entre eux. Chacun relate le plaisir à prendre part à cette démarche par le geste qui les engage physiquement et personnellement, dans une action « qui [les] dépasse ». Ces gestes par lesquels il devient possible d'inscrire durablement sa trace sur la terre semblent fondateurs, à la fois pour l'individu qui a fait le choix de s'engager dans une nouvelle voie mais aussi aux yeux du groupe. Les plantations se font souvent ensemble «parce que ça donne plus de sens », «ça engage », parce que «ça nous rapproche » ou encore parce que « ça nous permet d'être compris ». Le lien social se trouve ici impliqué dans une pratique agricole ordinairement esseulée. Les groupes réunis, appelés à participer, sont significatifs des réseaux et du parcours de vie de chacun des agriculteurs (chercheurs, élèves, amis, voisins, pairs, famille). Il s'agit d'acter collectivement, dans l'espace et dans le temps, le changement de direction opéré. Dans le cas de participation des proches de l'agriculteur, le temps familial lui-même est empreinté (et la mémoire de la famille). Amené à «grandir », l'arbre saura accompagner la succession des générations des hommes.

$\mathrm{Au}$ moment de la réalisation du chantier, étape particulière de l'élaboration paysagère, l'« habiter » tient lieu d'évènement spatialisé et daté, fait de formes et de gestes qui fondent un morceau de mémoire collective. « La première pierre, hop ! c'est un arbre » raconte Séverine.

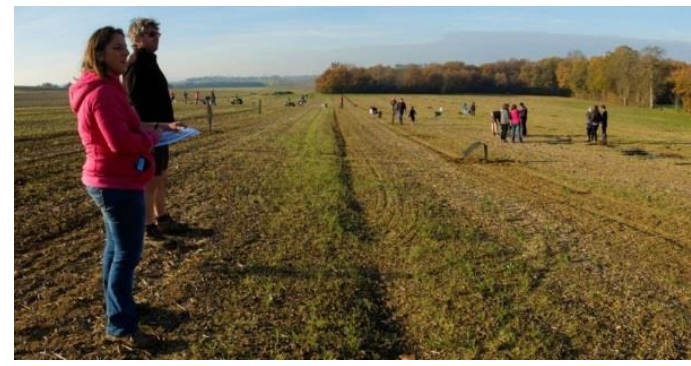

Figure 14. Chez Séverine et Jeff, le jour des plantations avec les proches et autres volontaires. (c) privée, à l'agricultrice 
Les arbres sont en terre, protégés par des gaines, arrosés parfois pour le « démarrage ». La bande enherbée à leur pied a été semée et devra être entretenue. Tout est en place. Commence le temps long. La croissance progressive des arbres accompagne la succession des cycles annuels. Nous pourrions à cette étape nous intéresser aux intercultures, à l'organisation du parcours des animaux (comment détourer les arbres, parquer la nouvelle parcelle ?) car ces choix animent différemment les paysages saisonniers. Nous pourrions aussi nous attacher à observer la façon dont les ligneux sont «conduits », formés - ou conformés - car ces pratiques déterminent autant les esthétiques que les productions possibles de la parcelle. Mais pour l'heure la taille constitue avant tout l'occasion de se rapprocher des arbres, l'un après l'autre, elle est un autre geste significatif et partageable qui se met en place. Elle se constitue année après année en rituel souvent considéré comme un temps pour soi, pris sur le temps libre (en dehors du temps agricole), parfois partagé en couple. C'est donc peutêtre moins au niveau du système de ferme qu'à celui de son récit qu'il $\mathrm{y}$ a, durant les premières années, d'importants changements. Le projet est là, manifeste mais poussant lentement. " Pourquoi ? Comment? » sont des questions indirectement posées à celui qui a fiché de gaines plastiques noires sa « plus belle parcelle !». Le projet semble avoir besoin des explications de l'agriculteur pour exister tout autant que ce dernier veut raconter son projet. Dans le film-recherche qui accompagne la thèse, on entend Séverine (l'éleveuse de volaille) qui reçoit des visiteurs sur sa ferme : « ... ça n'est pas qu'un verger, y a 400 arbres de plantés, et sur les 400 y en a seulement 30 qui sont des fruitiers greffés. Après, tout ça ce sont des érables, ça va faire une allée d'érables. Y a des chênes, y a des tilleuls, y a des frênes, y a des merisiers, y a des arbres qui vont devenir plus ou moins grands, qui poussent plus ou moins vite. Et on a choisi des essences qui permettaient de donner à manger aux poules : genre, les merisiers ils font des petites merises : bam, ça tombe au sol, et la poule elle les mange! ». Envie et besoin de traduire un paysage latent qu'elle seule a dans la tête, les arbres de Séverine cristallisent l'occasion de partager son nouveau métier et son application à réinventer son quotidien et le visage de la ferme de sa belle-famille.

Observons que durant ce temps long, continu et indéfini de la gestion de la parcelle plantée, par les cycles agricoles, au fil du quotidien, des rencontres et de l'expérience renouvelée de l'agriculteur, il émerge de nouvelles projections du paysage.

\section{Étape 6. Projection et transmission.}

Lucas est paysan-brasseur en agriculture biologique. Depuis qu'il a planté il s'est aperçu non pas seulement qu'il avait 300 arbres dans sa terre mais 10 parcelles au lieu d'une seule (figure 15). L'agroforesterie mise en place est devenue cette nouvelle trame qui remet maintenant en jeu les possibilités de son système de ferme : "Ça l'a agrandi et ça fait autant de champs et on peut imaginer avec du tout petit matériel 'Et si je faisais ça? ... De l'orge et puis ça: du pois-chiche, et puis ça et puis tiens là je fais un peu de patate'. Moi j'ai pas de bête mais par la suite, on sait jamais, il pourrait y avoir un troupeau. Mais on nourrit combien de famille? On peut en nourrir beaucoup... C'est là où je me dis on est tout seul sur nos immenses surfaces ... mais si on ramenait du monde y a moyen de faire beaucoup de choses. ». Il est «possible d'imaginer ». De vastes questions sont engagées comme celles de l'évolution du nombre d'actifs sur la ferme, la diversification possible des productions, des qualités d'espaces et de ressentis dans ces lieux : "Là on sera sur quelque chose de totalement différent, y aura beaucoup moins de lumière, mais plus d'ombre, pour l'été ce sera très bien. Peut-être que dans 20 ans pour le pastoralisme ce sera beaucoup mieux ce genre de parcelle. Mes voisins ils feront peut-être plus de foin. Là il y aura peutêtre quelque chose à brouter, voilà. Alors ça ne sera peut-être pas des moutons, ce sera des chèvres, je ne sais pas, on va voir. Et puis, au niveau des vents, tout ça ira mieux. Et je pense il y aura toujours une bande au centre, qu'on pourra continuer de faire en céréales. ...Moi là je vais vers la 
miniaturisation : plutôt que d'avoir toujours plus gros on va aller vers de plus petits tracteurs, de plus petits outils, ce qui va complètement à l'encontre de ce qu'on voit partout. Et puis même si on ne cultive pas là, je me dis un troupeau là-dessous sera très bien, il sera très très bien. ". Le paysage agroforestier serait ainsi le tremplin à une révision profonde de nos modes de production comme de nos modes d'habiter.

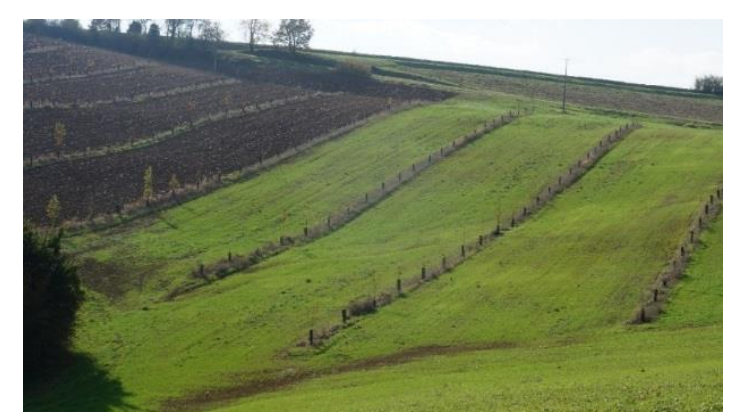

Figure 15. Chez Lucas, les « 10 nouvelles parcelles ». () Mathilde Rue

Parallèlement à la projection renouvelée du devenir des parcelles, nous avons pu relever l'intérêt des agroforestiers pour la transmission des parcelles. Puisqu'ils l'ont fait «pour après », elles prennent valeur de leg. Plus qu'à donner en héritage, les parcelles sont à confier. Notamment dans le cas où il n'y a pas d'enfants repreneurs, nous avons pu entendre cette crainte : "Je ne sais pas ce que ça va devenir. Des fois je me dis, holala, s'il fallait partir, si c'est le voisin qui récupèrerait ça, première chose qu'il ferait, il ferait tout sauter. " Franc rassure Chantal : "Si on part de là, on va s'arranger pour ne pas vendre à des abrutis. ». Ici est rendue évidente la préoccupation pour que la transmission se fasse dans une continuité de valeurs et de pratiques. Car pour que ça marche, pour que leur geste « rende à la nature » il faut qu'il dure, que les arbres restent. Ces récits mettent en exergue la fragilité des projets. D'une part, le départ anticipé ou la reprise incertaine de la ferme, la mortalité des arbres, les conséquences de modes de gestion inadaptés ou encore un mauvais développement des végétaux sont possibles. Mais il y a aussi l'inconnu paysager qui rend difficile la description des paysages et des conditions futures initiées au présent. «Je ne sais pas du tout ce que ça va donner cette affaire » déclame Pierre, au terme du récit qu'il fait de son projet lors de notre discussion. Dans cette parole est évoquée à la fois l'incertitude de l'exploitation finale des arbres (la récolte du bois : quand, comment, par qui, pour quels gains ?) voire de leur maintien jusque-là, mais il est aussi avouée la difficulté à visualiser le paysage à venir. Nous avons vu que la parcelle agroforestière est le résultat d'une hybridation de paysages. Elle est une configuration originale et située qui n'a pas de réplique ! Le paysage de l'agriculteur n'existe donc pas ailleurs que chez lui.

Sur le secteur d'enquête, parmi les 16 fermes, deux d'entre elles ont accueilli un projet agroforestier il y a plus de 30 ans (soit bien avant les 14 autres agriculteurs qui ont planté à partir de 2011). Les contextes étaient très différents. Le premier des deux porteurs de projets, Roland, s'est détourné de ses parcelles intraparcellaires au fil des années. Elles lui ont été plantées par les services de la DDA à l'occasion d'un programme subventionné. Roland a cependant poursuivi des actions de plantations en de multiples endroits et sous d'autres formes. Cette évolution-ci pose le problème de la non-appropriation des parcelles sous réglementation trop «fermée » en même temps qu'elle confirme la motivation fondamentale de ces agriculteurs à planter des arbres, coûte que coûte.

La seconde occurrence de dimension historique, menée par Wim, a elle joué avec les possibilités qu'offrait la règlementation du Fond National Forestier. Au cours de plusieurs années de mise au point et de négociation, l'agriculteur est parvenu à planter selon les modalités qu'il recherchait. Au contraire de 1'expérience de Roland, Wim poursuit actuellement une gestion très impliquée de ses 
parcelles agroforestières : il améliore, replante, expérimente et recompose à l'intérieur d'ellesmêmes.

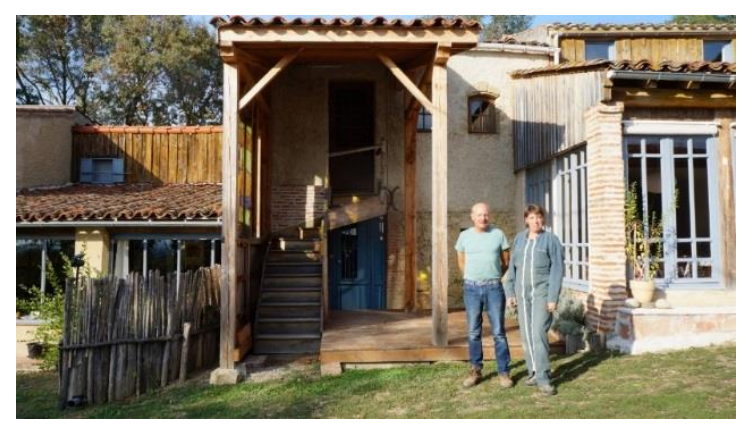

Figure 16. Wim et l'une de ses filles devant chez lui. (c) Mathilde Rue

Sur l'image (figure 16), Wim est avec une de ses filles, devant sa maison : une ancienne ruine qu'il avait restaurée il y a 30 ans avec son épouse, en même temps qu'ils plantaient les 15 ha acquis et répartis tout autour. Wim parle d'une installation « rurale » plus qu'agricole. Aujourd'hui, sa fille a sa maison à côté, elle a repris la ferme et monté son activité, elle propose des produits laitiers de brebis en vente directe. Si nous déportons notre regard sur le territoire qui les entoure, il est saisissant de constater l'écart avec les ilots voisins. Ces derniers ont connu une toute autre évolution: ils se sont agrandis et «désarbrés ». Le projet de l'«habiter» de Wim issu de ses expériences de vie en Israël dans une ferme collective et en Afrique du Nord, a donc tenu dans un contexte opposé sinon hostile. Cette persévérance, en tous cas cette persistance du projet démontre la force paysagère à l'origine de ces plantations-ci. Telle une importation paysagère, si vous vous y rendez, vous rencontrerez des ambiances, des produits agricoles et des idées originales. Car les gestes, la façon d'arpenter, le quotidien, toute l'organisation et ce qu'ils racontent de leur ferme est particulier à eux et fonde un habiter singulier.

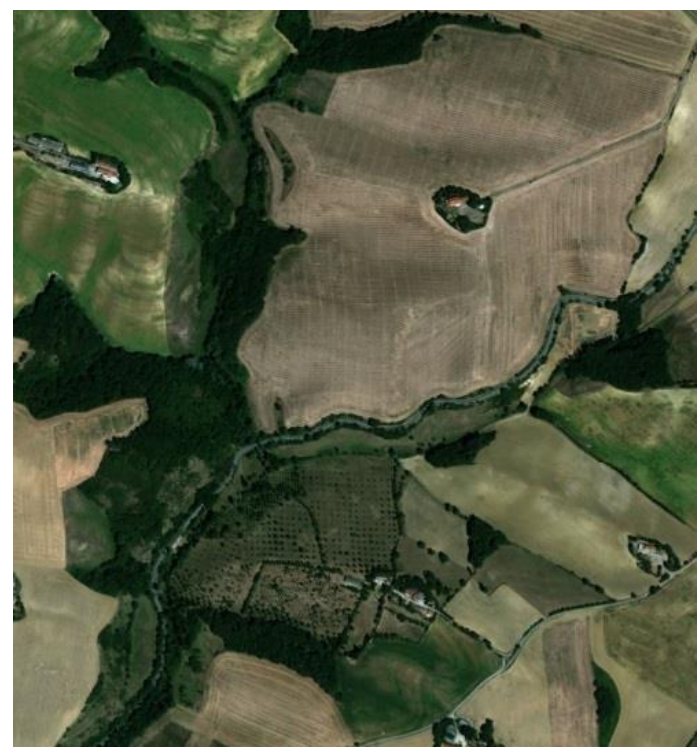

Figure 17. L'ilot de Wim (en bas) et ceux des voisins (C) Mathilde Rue 

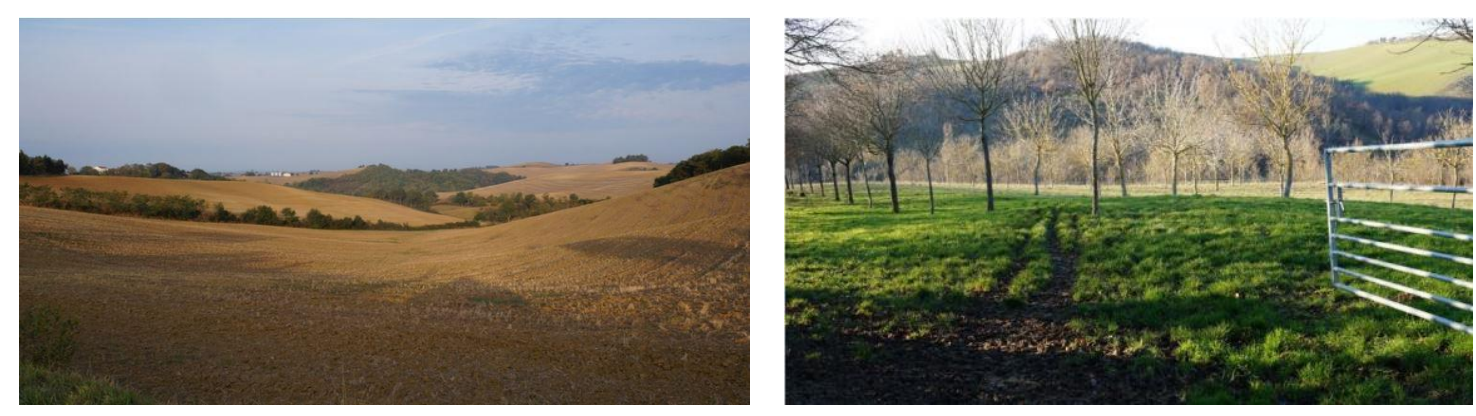

Figure 18. Vue d'arpentage de l'îlot voisin à celui de Wim. () Mathilde Rue Figure 19. Vue d'arpentage de lîlot de Wim. (c) Mathilde Rue

\section{Conclusion}

«Interroger l'habiter, c'est interroger ce qu'il en est pour les hommes de leur monde, du monde qu'ils ont édifié au cœur de l'espace et du temps, dans lequel ils ont ordonné leurs existences individuelles et collectives, mais aussi dans lequel, tout simplement, ils vivent. » (Besse, op. cit., p7). L'agroforesterie comme manifestation de l'habiter donne en effet à comprendre autant les rêves que le quotidien plus prosaïque de ces agriculteurs de la Haute-Garonne.

A l'occasion de l'élaboration paysagère, nous pouvons mettre en évidence différentes dimensions de «l'habiter » que le processus intègre. Habiter c'est d'abord trouver un lieu à soi, où l'on est bien, et en faire sa «base » (Levinas, 1986, p120). Une fois que ce centre est connu, le reste à partir de lui peut s'organiser. Il s'agit de faire sa bulle (le microcosme), son « nid » à la mesure de ses gestes, de ses mouvements, de ses activités et objets (Bachelard, 1957, p 100-101). Pour se faire, les agriculteurs organisent leurs espaces. Ils inscrivent des marques dans le sol, ils s'écrivent, individuellement d'une part mais aussi collectivement. Leur trace, si elle est nouvelle, vient en poursuivre d'autres, l'agriculteur s'ancre dans une généalogie de l'espace (Bruno et l'espace ombragé des vaches de son enfance). Habiter s'édifie avec les autres, en famille, avec les voisins, avec le territoire. Car elle instaure des moments partagés, la parcelle agroforestière peut être envisagée comme l'installation d'une zone de contact : un seuil entre la ferme et le reste. D'abord par l'évènement du chantier de plantations puis du fait de son architecture qui, sans doute se prête à une mise en forme spatiale et sémantique de l'hospitalité. Au même titre que les entrées ou les auvents, les parcelles tramées d'arbres seraient des «espaces intermédiaires» précipitant «l'occasion de concilier des mondes qui se côtoient» (Hertzberger, 2010, p 50). Cette écriture «en puissance " s'accompagne de temps de latence durant lesquels le paysage est raconté par l'agriculteur et donné à être imaginé pour le visiteur. Travail de la main (« aménager », «ménager ») constitué de gestes incarnés qui deviendront plus grands, l'attention est à faire pousser ce «cela pourra être ». Car habiter c'est, enfin, préparer la suite après sa propre habitation, et donc confier l' écriture de sa propre existence.

Dans ces processus de projet, il y a bien un «trajet-de-paysage » (Berque, 1984), soit des jeux d'empreintes-matrices qui interviennent entre les expériences paysagères des agriculteurs et le projet agroforestier engagé. La parcelle se dessine dans cette sédimentation de paysages diachroniques et déterritorialisés (Deleuze et Guattari, 1972) et notre travail filmique cherche à rassembler à cet effet les ingrédients de la projection de l'agriculteur (points de vues, espace-temps convoqués ou « strates paysagères ») afin de donner à comprendre, pour le spectateur les «lignes de fuite » (Ibid.) que créent les paysages agroforestiers.

Au regard de la diversité des projets fondés que nous avons exposés, nous pourrions dire que ce 'mode de faire' le paysage est pluriel. Mais est-il singulier à la situation de la ferme ? L'effort projectif des agriculteurs (essayant de composer avec les techniques, les règlementations, les (C) 2019 ISTE OpenScience - Published by ISTE Ltd. London, UK - openscience.fr 
compétences et les outils disponibles) n'est pas sans évoquer l'aventure du « projet de paysage » des concepteurs-paysagistes. Telles deux voies de transformation du territoire, travaillant par portion l'habitabilité du monde, la première serait «habile» et habitante, la seconde « experte » et extérieure. Chacune tenterait d'initier des relations paysagères actives, habitantes - pour soi (le concepteur-agriculteur dans son territoire) ou pour les autres (le paysagiste invité à transmettre un paysage à partir d'un site qui lui est inconnu (Kéravel, 2015)). Le souci écologique en donne la commune mesure au sens où, ces deux processus s'intéressent au sens porté par les formes choisies, aux rapports d'échelle corps-espace ou encore au temps long.

\section{Bibliographie}

Bachelard, G., La Poétique de l'espace, Paris, PUF, 1957.

Besse, J-M., La nécessité du paysage, éditions Parenthèses, 2018.

Besse, J-M., Le Goût du monde. Exercices de paysage, Arles, Actes Sud, 2009.

Besse, J-M., Habiter : un monde à mon image, Flammarion, 2013.

Berque, A., "Paysage-empreinte, paysage-matrice : éléments de problématique pour une géographie culturelle », Espace géographique, T. 13, n 1,1984 . pp. 33-34, URL :

www.persee.fr/doc/spgeo 0046-2497 1984 num $13 \quad 13890$.

Berque, A., " Milieu et motivation paysagère », Espace géographique, T. 16, n 4, 1987, pp. 241-250, URL : www.persee.fr/doc/spgeo 0046-2497 1987 num 1644266.

Convention européenne du paysage, traité du conseil de l'Europe, 20 octobre 2000, Florence.

Corbin, A., L'Homme dans le paysage, Paris, Les Éditions Textuel, 2001.

Datar, Paysages photographies. La mission photographique de la Datar. Travaux en cours, 1984-1985, Paris, Hazan, $1985,517 \mathrm{p}$.

Deleuze, G., Guattari, F., L'Anti-CEdipe. Capitalisme et schizophrénie, Paris, Éditions de Minuit, 1972.

Dupraz, C. et Liagre, F., Agroforesterie. Des arbres et des cultures (2010), Paris, France Agricole, 2013.

Henry, D., «Entre-tenir la montagne : paysage et ethnogéographie du travail des éleveurs en montagne pyrénéenne : hautes vallées du Gave de Pau, de Campan et d'Oueil-Larboust », thèse de doctorat, université Toulouse le MirailToulouse II, 2012.

Hertzberger, H., Leçons d'architecture, trad. L Biétry, Gollion, Infolio, 2010.

Kaufmann, J.-C., L'Entretien compréhensif, Paris, Armand Colin, 2013.

Kéravel, S., Passeurs de paysages, le projet de paysage comme art relationnel, Éd. MétisPresses, 2015.

Levinas, E., De l'existence à l'existant, Paris, Vrin, 1986.

Roger, A., Court Traité du paysage, Paris, Gallimard, coll. « Bibliothèque des sciences humaines », 1997.

Rue, M., «L'agroforesterie intraparcellaire au cœur d'une élaboration paysagère menée par l'agriculteur », Projets de $\begin{array}{lllll}\text { paysage, } & \mathrm{n}^{\circ} & 19, & \text { décembre } & 2018,\end{array}$ http://www.projetsdepaysage.fr/fr/l agroforesterie intraparcellaire au c u0153ur d une laboration paysag re men e p ar I agriculteur. 